EVS26

Los Angeles, California, May 6 - 9, 2012

\title{
Routing EV Users Towards an Optimal Charging Plan
}

\author{
Sandford Bessler ${ }^{1}$, Jesper Grønbæk ${ }^{1}$ \\ ${ }^{1}$ FTW Telecommunication Research Center, Donau-City 1, 1220 Vienna, Austria, bessler@ftw.at
}

\begin{abstract}
In this work we address the efficient operation of public charging stations. Matching energy supply and demand requires an interdisciplinary understanding of both the mobility of electric vehicle (EV) users and the load balancing mechanisms.

As a result of existing mobility studies, we propose in this work a routing service for searching and reserving public charging spots in the neighborhood of a given destination. When comparing the search results for direct drive with those for a multimodal route (using driving, walking and public transport) in an urban environment, we obtain for the latter significantly more charging options in particular at low e-mobility penetration levels, at a cost of slightly longer trip duration.

Further contributions address the schedule optimization, that, due to the proposed distributed architecture, can be performed independently at each public charging station. We formulate an integer program for the controlled charging and compare results obtained both with the exact and with a greedy heuristic method.
\end{abstract}

Keywords: e-mobility, EV routing service, multimodal route optimization, charging station, controlled charging, power flow calculation

\section{Introduction}

A well studied scenario for charging electric vehicles (EV) is charging overnight at home. This scenario alone, however, fails to address certain significant user groups such as residents of urban areas without own garage, vehicle fleets, vehicles with higher mileage, etc. These users are all dependent of the existence of public charging stations (PCS). Using a PCS poses however two problems for the user: a) to find a PCS that matches the mobility needs and $b$ ) the found PCS must be available in terms of energy and parking space in the desired charging period. We address both interdependent problems in this work, that has been conducted within the KOFLA project [1]

As the vehicular mobility can shift energy charging energy very quickly from place to place, the main idea we follow in this work is to plan resources in advance that contribute both to user satisfaction and to the service performance of the grid operator.

Recent mobility studies [2],[3] reveal that EV charging must be subordinated to the mobility goal or activity and not viceversa. As a conse- quence, users should plug-in their EVs in walking distance of their destination. Partial charging is acceptable, if the stay duration is limited.

Based on these assumptions, we have defined a query and reservation protocol between an EV and a routing service that enables the user to: a) query availability of charging stations anytime in advance, b) reserve a time slot for charging at an available charging station, and c) be notified, when a charging point becomes available. The protocol runs over a wireless channel available in a cellular network GSM/UMTS/LTE, but in the future it could be integrated in the ITS service ecosystem, as proposed currently in ETSI EV notification draft [18].

The routing service is designed to serve a geographical region and represents a broker between EV users and energy providers. The broker entails the best PCS match in terms of availability of resources required for charging. It further considers user preferences such as location convenience, price importance, preferred energy provider, etc.

There are two parameters in the user provided information (see Table 1) that help to route the user and reserve resources at a selected PCS: the time 
window, i.e. estimated arrival and leave time, and demand, i.e. the maximum amount of energy needed. Whereas the arrival time and energy can be estimated by the vehicle navigation and the battery management system, the stay duration has to be provided by the user (but it could also be infered from the activity type).

Table 1: Parameters of the query message

\begin{tabular}{lc}
\hline Parameter & value \\
\hline timestamp & \\
destination (next stop) & coordinates \\
expected arrival time & \\
expected departure time & \\
expected State of charge (SoC) & \\
may use public transport & yes/no \\
charging rates supported & slow/normal/quick \\
price importance & high, low \\
waiting importance & high, low \\
walking distance importance & high, low \\
renewable importance & high, low \\
payment means & card name, null \\
\hline
\end{tabular}

For this scenario, we introduce a decentralized system architecture, where each PCS is able to schedule and control the charging of individual EVs allocatd to it. In this way the architecture supports a new stakeholder: the charging station owner. This provider has the freedom and incentive to setup charging stations and sell the park \& charge service in conjunction with added value services like loyalty programs, public transportation (see discussion in [4]).

Figure 1 shows schematically the proposed architecture: the routing server dispatches the reservations on the basis of the rough availability of resources at certain selected public charging stations. Each PCS sends load status updates both to the routing service and to a LV-grid agent, which has the task to calculate periodically the feasible charging load (available power) for each PCS, using a power flow model. In the example in Figure 1 , PCS A and B are in the same LV-grid, whereas PCS C is in a different LV-grid.

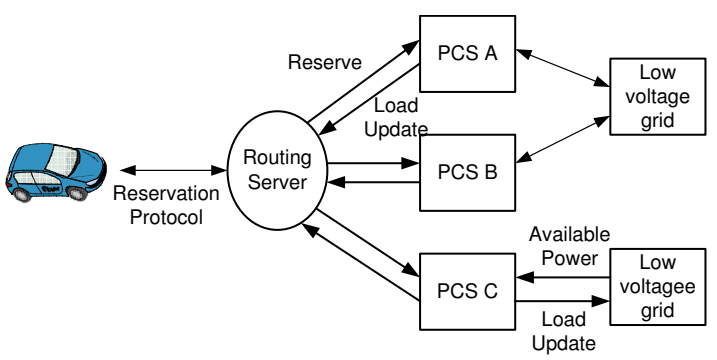

Figure 1: System architecture: charging load balancing is performed through routing the requests to different PCSs and through optimized activity scheduling in the PCS.

In the rest of the paper, we focus on two mechanisms that would drastically improve the avail- ability of charging resources at a PCS. In section 2 we evaluate the park, charge \& ride option and in Section 3 we study the optimal allocation of resources at a specific PCS.

\section{Multimodal Routing}

Multimodal routing covers the aspect of including different means of transportation in a route. Multimodality is relevant for park-and-ride scenarios where drivers park in the perimeter of the city and continue to their destination using public transport. Multimodality with public transport can also be combined with e-mobility charging to increase the access to more charging stations while still ensuring end-to-end mobility. Increased charging station availability is important in early low penetration scenarios. Further, identifying several charging station options can be beneficial to increase the possibility of identifying a charging station with free resources.

In this section, we will shortly revisit our previous work from [19], which introduces a multimodal routing scheme and a preliminary analysis on gains in charging station availability. In this work, the preliminary analysis is extended to assess the travel time impact of multimodal routes under different road traffic conditions.

\subsection{Multimodal Routing Heuristic}

The multimodal routing heuristic proposed in our previous work [19] involves the modalities of EV-driving, public transport and walking. The objective of the heuristic is to identify a set of charging stations which are adjacent to public transport stops that can provide user-mobility to the final destination. The heuristic is based on a set of presumptions: 1) An EV-user can accept to walk maximally $T_{\text {walk }}$ seconds between two points on a route i.e. from charging station to public transport stop and from a public transport stop to the destination, 2) The user can accept to spend maximally $T_{p t}$ seconds in public transport transit, 3) The desired EV driving range is limited to $E V_{\text {range }}$ which enables for instance to satisfy low battery EV range constraints. The heuristic is realized as follows:

Off-line initialization: All charging stations within $T_{\text {walk }}$ seconds of a public transport stop are identified.

Online processing (at route request):

A. Identify the public transport stop closest to the destination $P_{d s}$ (destination stop), which is within $T_{\text {walk }}$ walking distance. If none are found, return an empty set.

B. Identify public transport stops $P_{c s s}^{i}, i=$ $0 \ldots N$ (charging station stop). $\stackrel{N}{N}$ corresponds to the amount of stops that can be reached within $T_{p t}$ seconds from $P_{d s}$ (assuming symmetric of public transport routes). The travel time to each station can be calculated from a shortest-path-tree [14]. 
Based on $P_{c s s}^{i}$, look-up all charging stations $C S_{\text {all }}^{j}, j=0 \ldots M$ where $M$ are the amount of charging stations within walking distance of the stops in $P_{c s s}^{i}$.

C. Of the charging stations $C S_{\text {all }}^{j}$ filter out irrelevant charging stations based on $E V_{\text {range. }}$ In the following evaluations, charging stations are considered in a radius defined by the distance from the EV to the destination stop +2000 meter to avoid excessively long routes.

D. For each remaining charging station, calculate two routes: 1) driving from the current location of the EV to the charging station and 2) public transport from the charging station to the destination. These routes are merged to a joint multimodal route.

E. The routes calculated for each charging station in D. are returned in a final set $C S_{\text {options }}^{k}, k=0 \ldots P$ to the EV-user for a final selection. $P$ is the total amount of reachable charging stations returned.

To evaluate the proposed mechanism, it has been realized using open source route calculation tools. Graphserver [20] is applied to derive the public transport and walking routes whereas Gosmore [21] provides vehicle routes. An evaluation scenario has been constructed from the $\mathrm{Vi}$ enna region which represents a large city with a strong public transport network. For the evaluation, only the subway system is considered. It covers the major parts of the city region and also a majority of all public transport trips conducted in the region (64\% [24]). Geographical data are obtained from OpenStreetMap.org [12] and public transport data from "Wiener Linien".

Results on charging station reachability and impact on total travelling time have been obtained by generating trips in a $16 \mathrm{~km}$ by $16 \mathrm{~km}$ area depicted in Figure 2. Note, the term reachability is used instead of availability as the scope of this work only is to consider if a charging station can be reached and not if it is free or occupied. A trip consists of a starting point and a destination. For each trip three routing schemes are calculated: 1) a direct route which defines the driving time directly to the destination (without charging), 2) a charging in destination vicinity route, where a charging station is within $T_{\text {walk }}$ seconds from the destination, and 3) a multimodal route via a charging station and public transport. A multimodal and a direct route for an example trip is provided in Figure 2. To generate and compare different trips, independent start and destination locations are generated. Start locations are randomly generated (uniform) all over the region to simulate trips starting in the city as well as the surroundings. The destinations are randomly chosen from a database with points-ofinterest (from data.wien.gv.at containing schools, restaurants, hotels, sport centers, etc.) to ensure commonly relevant destinations.

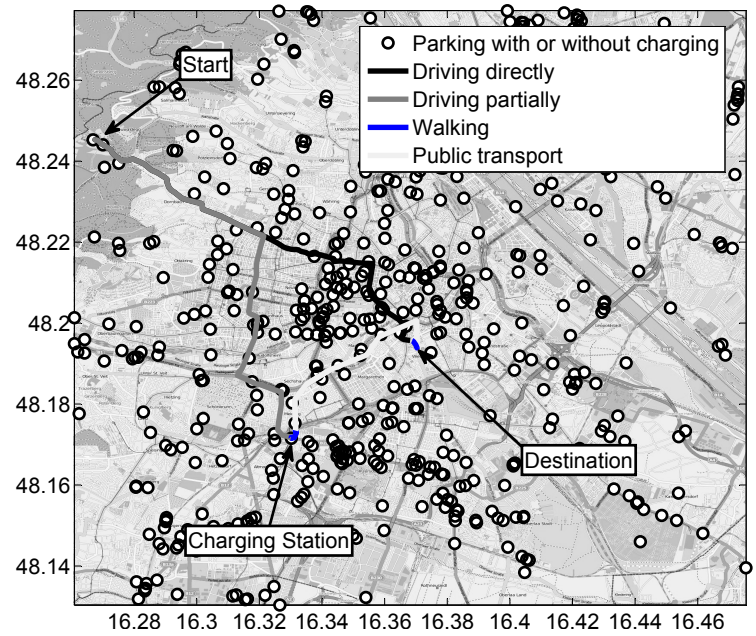

Figure 2: Example trip from start to destination by direct driving without charging, driving to a charging station in vicinity of the destination and travelling via public transport.

To assess the impact of multimodality in different degrees of electrified public parking places, three different $\mathrm{EV}$ penetration levels have been compared: $10 \%, 50 \%$ and $100 \%$. The penetration levels are controlled by the parameter $p_{C S}$, which describes the probability that a public parking place has been electrified. For the evaluation scenario, public parking places have been obtained from the OpenStreetMaps data leading to a total of 568 parking places. These are also depicted in 2.

Table 2: Main parameters used in the simulation of routing schemes.

\begin{tabular}{lc}
\hline Parameter & Value \\
\hline Trips evaluated & 484 \\
Walking speed & $5 \mathrm{~km} / \mathrm{h}$ \\
Trip start time & 09:00:00 (weekday) \\
$T_{p t}$ & unlimited \\
$T_{\text {walk }}$ & $300 \mathrm{~s}$ \\
\hline
\end{tabular}

From the analysis presented in [19], significant gains have been shown in terms of charging station reachability. The previously obtained results are summarized in Table 3 using the values of Table 2 comparing the destination vicinity charging scheme to the multimodel routing scheme. The results show the fraction of trips where at least one $(P>0)$ or at least $3(P \geq 3)$ charging stations are reachable. Identifying several reachable charging stations is clearly necessary when considering that for a given charging station no free capacity may be available.

For both results sets, significant benefits of the multimodal scheme can be demonstrated; especially in low penetration scenarios. For more details on this analysis interested readers are referred to [19]. 
Table 3: Results of reachability of one or more charging stations on a trip.

\begin{tabular}{|l|l|l|l|}
\hline CS set & $p_{C S}=0.1$ & $p_{C S}=0.5$ & $p_{C S}=1$ \\
\hline$P>0$ & $8.7 \%$ & $39.3 \%$ & $62.6 \%$ \\
& $\mathbf{4 0 . 5} \%$ & $\mathbf{6 1 . 0} \%$ & $\mathbf{7 4 . 2} \%$ \\
\hline$P>3$ & $0.0 \%$ & $1.9 \%$ & $11.0 \%$ \\
& $\mathbf{3 2 . 9} \%$ & $\mathbf{3 5 . 7} \%$ & $\mathbf{4 1 . 1 1} \%$ \\
\hline \multicolumn{3}{|c|}{ Destination vicinity charging, Multimodal } \\
\hline
\end{tabular}

\subsection{Impact of Traffic Conditions}

In the following, a quantification of the impact of the multimodal routes is made compared to destination vicinity charging routes in the Vienna city scenario. The quantification focuses on the change in average trip travel time between the two schemes considering different road traffic conditions. This enables to clarify the potential advantages of the subway based transport, which is clearly not affected by varying road traffic conditions. For the study, two extremes are considered: perfect low traffic conditions and during rush hour.

To model the road traffic conditions a simplistic model is applied. The model separates the road segments of the studied region into the classes of city roads and motorways. Each class is parametrized by an average speed. This simplistic model is in correspondence with existing traffic information systems for the Vienna region allowing for a direct mapping of existing statistics [23]. The traffic conditions are modelled as average speed conditions for all vehicles in the road network. As such the analysis does not take into consideration potential congestion scenarios in individual trips. Average speed parameters for city roads have been obtained from the work in [22]. The authors describe an ITS system for online collection of traffic data based on taxis with GPS. The system has been deployed in Vienna to enable realistic average speed estimates under the different conditions. Also based on GPS data, the work of [23] similarly presents average values for motorways in the Vienna region. A summary of the utilized parameters is provided in Table 4.

Table 4: Average speed according to traffic conditions and road type.

\begin{tabular}{|l|l|l|}
\hline $\begin{array}{l}\text { Traffic } \\
\text { conditions }\end{array}$ & City roads & Motorway \\
\hline Ideal & $30 \mathrm{~km} / \mathrm{h}$ & $85 \mathrm{~km} / \mathrm{h}$ \\
\hline Rush hour & $20 \mathrm{~km} / \mathrm{h}$ & $58 \mathrm{~km} / \mathrm{h}$ \\
\hline
\end{tabular}

The classification of road segments into respectively city roads and motorways in the region of Figure 4 has been made from maxspeed values available in the OpenStreetMap data. Road segments with a maximum speed of $80 \mathrm{~km} / \mathrm{h}$ or above are classified as motorway.

Utilizing the evaluation approach introduced in Section 2.1 and the values from Table 4 complete route traveling times have been calculated.
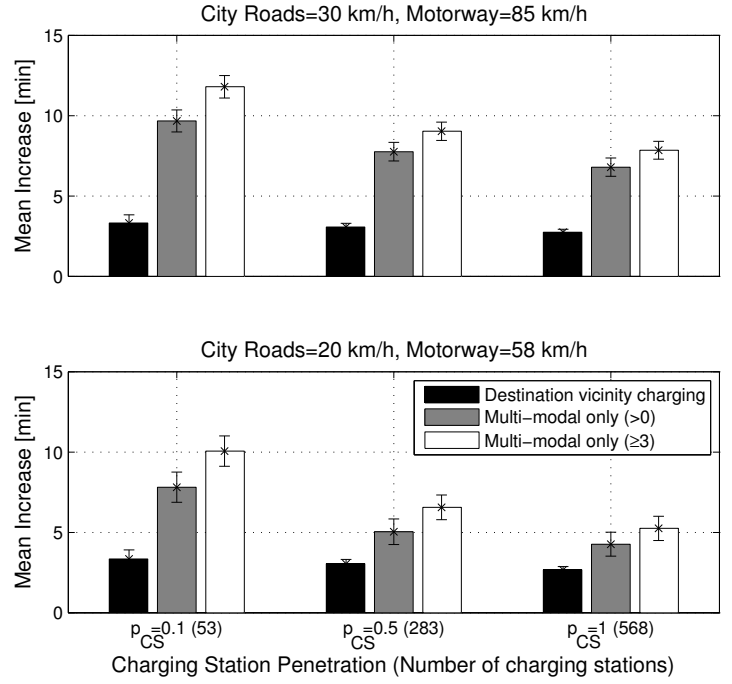

Figure 3: Comparison of the overall trip time increase compared to driving directly to the destination.

Note, that for this evaluation $T_{p t}$ is set to unlimited to not sort out any routes where driving primarily with the subway could be the fastest approach. Figure 3 presents the mean increase in travel time for the two routing approaches under different charging station penetration levels. The increase is calculated in relation to the direct route to the destination (without charging). The results suggest under ideal road conditions that the difference in increase between the destination vicinity charging and the fastest multimodal route (grey bar) are between 4 and 7 minutes depending on the penetration level. Also, it can be observed that the mean travel time does not increase significantly when considering using one of the three fastest multimodal routes (the white bar). Under rush hour traffic conditions, it can be observed that this difference is decreased to between 1 minute (for $P_{C S}=1$ ) and 5 minutes for low penetration scenarios.

Which increase in travel time EV owners subjectively are willing to accept is up to future usability studies to clarify. It is, however, clear that the multimodal scheme offers a significant improvement in the reachability of charging stations. Further, our results suggest that in medium to high penetration scenarios and rush hour conditions the mean travel time increase is in the order of a few minutes. Overall, the results are encouraging and future work must establish how the increased reachability of charging stations can be applied to improve overall charging availability and potentially geographical balancing of grid load.

\section{Controlled Charging at the Charging Station}

With the increase of EV penetration, controlled charging, i.e. the coordination of charging time slots will become indispensable both in residen- 
tial areas such as apartment blocks with electrified parking lots, and public charging facilities. A controlled charging strategy schedules the charging jobs in such a way that it reduces load peaks caused by users that plug-in their cars approximately in the same time. In contrast to other works on recharge scheduling [8],[9],[10], our model uses as key parameter the time window corresponding to user's estimated parking start and end time. Further required are: the amount of energy needed to fill up the battery and the charging rates (in $\mathrm{kW}$ ) supported by both the vehicle and the charging station.

\subsection{Problem Formulation}

For the problem we want to solve there is given a time horizon of $\mathrm{T}$ discrete periods, a number of $\mathrm{M}$ charging points and a number of $\mathrm{A}$ activities, so that each needs one of the $M$ parking places for a duration time window (earliest,latest), and 2) a power resource for a certain duration which depends on the selected charging speed $s p_{k}$ (from a discrete set). The solution has to determine the start charging time for each activity, the allocation to a charging point, such that the total power consumed in each time period does not exceed the planned value, and such that a certain profit objective function to be explained below is maximized.

Note that we have to deal with two types of resources: parking place during the whole time window, and power limitation during the charging period. If we make the assumption that the charging points are identical (machines), we can split the problem into two sub-problems:

1. since the machines are identical, we allocate first the activity time windows to the charging points. This problem is similar to scheduling classes to classrooms, and can be solved optimally by a greedy heuristic (increasing starting time rule). The intervals are the activity time windows and cannot be shifted. The resulted allocation of activity to charging point allows us to address the second subproblem as a one machine problem.

2. this is a bandwidth or resource allocation problem over time intervals, or RAP) which is NP-hard, since it can be reduced to a knapsack problem if the time windows are set to the intervaal $[0,1]$. The specific problem which we denote EVRSTW (electric vehicle recharge scheduling with time windows) requires that the charging takes place within the time window of the activity, has different speeds (supported by both car and charging point), and is limited by total power available in each of the T periods.

In case the machines are not identical, i.e. slow and fast charging, the machine allocation can be still made under the following assumption: fast charging spot is an expensive resource, therefore is will not be used for slow charging, which leads to two disjoint activity groups. In the most general case, instances have to be created for each

\begin{tabular}{|c|l|}
\hline & Description \\
\hline$T$ & set of time periods $t \in T$ \\
$A$ & Set of charging activities \\
$M$ & set of charging points \\
$I$ & instances generated from activities A \\
$e_{j}, l_{j}$ & time window (earliest, latest) $j \in A$ \\
$s_{i}, f_{i}$ & start and finish time of charging , $i \in I$ \\
$d_{j}$ & energy demand of activity $\mathrm{j}$ \\
$P^{t}$ & total available power during $t \in T$ \\
$w_{i}$ & charging speed $i \in I$ \\
$v_{i}^{t}$ & parking period of instance $i \in I, t \in T$ \\
$c_{i}$ & completion degree of instance, one of C \\
$p_{i}$ & profit generated by instance $\mathrm{i}$ \\
$x_{i}$ & variable: $x_{i} \in\{0,1\}, i \in I$. \\
$k\left[s p_{i}\right]$ & cost for charging at speed $s p_{i}$ \\
\hline
\end{tabular}

Table 5: Summary of notation.

individual machine, making the problem very large.

In the rest of this section we assume the machines are identical, so that the allocation activitycharging spot has been done and focus only on the second subproblem, the RAP. The RAP problem is highly combinatorial, since the charging interval can be selected anywhere within the time window, can have different durations determined by the charging speed and the completeness criteria, leading to different profits for the same activity. In order to simplify the integer linear program (ILP) formulation, we generate from each parameter combination an instance $i$ and use one binary variable $x_{i}$ to denote that that instance is selected in the solution, see Bar-Noy et al [15] : for $j \in A$

for $m \in M$ (not identical $\mathrm{m}$ )

for $n \in C$

for $k \in S$

for $l \in\left[e_{j}, l_{j}-d u r\right], d u r=n d_{i} / k$

generate instance $\operatorname{inst}(i, m, n, k, l)$

end

end end

end end

The profit $p_{i}$ is defined as a weighted sum of two objectives: the completion degree $c_{i}$ and the cost factor $k\left[s p_{i}\right]$ of the charging speed (quick charging requires more expensive equipment and shortens the battery life). We set $\alpha=0.5$

$$
p_{i}=\alpha c_{i}+(1-\alpha) / k\left[s p_{i}\right]
$$

Problem EVRSTW:

$$
Z=\max \sum_{i \in I} p_{i} x_{i}
$$




$$
\begin{gathered}
\text { s.t. } \sum_{i \in I \mid a[i]=j} x_{i}=1, j \in A \\
\sum_{i \in I \mid t \in\left[s_{i}, f_{i}\right]} w_{i} x_{i} \leq P^{t}, t \in T \\
\sum_{i \in I \mid m[i]=m} v_{i}^{t} x_{i} \leq 1, m \in M, t \in T \\
x_{i} \in\{0,1\}, i \in I \\
\sum_{i \in I} x_{i} \leq 1
\end{gathered}
$$

The inequality (4) limits for each time slot $t$ the total power due to simultaneous activities to the available power $P^{t}$. The equality (3) makes sure that exactly one instance of each activity is selected.

\subsection{Heuristic Algorithm}

Since problem EVRSTW is NP-complete, a natural step would be to look for approximation heuristics that would performe satisfactorily in a large charging station.

A first selection has been the local ratio algorithm which belongs to a class of stack based heuristics [16], and has been proposed in packing problems to maximize bandwidth throughput [17], [15]. In our case the bandwidth corresponds to the charging power. For this type of problems, the algorithm can achieve a $1 / 3$ approximation, which gets better as the ratio between the charging power (speed) of individual activities and the total available charging power is smaller. The algorithm works as follows: the instances $I$ are first sorted in increasing order of their end (charging) times. In the first sweep we select an instance with minimum end time and decrease the profits of all instances that a) belong to the same activity, b) overlap with the selected instance. Instances with non-positive profits are removed, and the selected activity is pushed on a stack. When no activities remain unconsidered, in the second sweep activities are popped from the stack and those who violate the total power constraint (4) are deleted. Denote the selected instance $\tilde{i}$, then the profits of conflicting instances $p_{i}$ are reduced as follows: $p_{i}=p_{i}-\beta \bar{w}_{i} p_{i}$, where $\beta=2$. Other than in the exact algorithm, the amount of resource has to satisfy $0 \leq \bar{w}_{i} \leq 1$. The problem arises at the calculation of if $\overline{\bar{w}}_{i}$ if $w_{i}$ varies in the interval $\left[s_{i}, f_{i}\right]$. The conservative rule would be

$$
\bar{w}_{i}=\min _{t \in\left[s_{i}, f_{i}\right]} w_{i}
$$

Another rule would be to use the average over the interval. The algorithm and its implementation are described in [15].

\begin{tabular}{|l|l|l|l|l|l|l|}
\hline$|A|$ & $|M|$ & $P$ & $Z_{I P}$ & $t_{I P}$ & $Z_{H}$ & $t_{H}$ \\
\hline 20 & 16 & 30 & 13,5 & 11 & 9,9 & 9 \\
40 & 30 & 40 & 23,1 & 19 & 15,8 & 27 \\
80 & 48 & 70 & 40,8 & 37 & 35,4 & 132 \\
120 & 64 & 90 & 62 & 32 & 51,5 & 550 \\
160 & 80 & 150 & 93,9 & 20 & 74,7 & 1260 \\
50 & 32 & 40 & 25,1 & 18 & 20,2 & 45 \\
50 & 32 & 50 & 30,0 & 17 & 24,5 & 48 \\
50 & 32 & 60 & 32,3 & 17 & 26,9 & 55 \\
50 & 32 & 70 & 33,2 & 22 & 29,0 & 60 \\
50 & 32 & 80 & 33,9 & 18 & 28,6 & 62 \\
\hline
\end{tabular}

Table 6: Results for the integer problem (IP) and Heuristic $(\mathrm{H})$ : $\mathrm{Z}$ is the objective, $\mathrm{t}[\mathrm{s}]$ is the runtime, $\mathrm{P}[\mathrm{kW}]$ is the available power (here constant).

\subsection{Computational Results}

We first have evaluated the integer program EVRSTW and the local ratio algorithm in terms of running time and accuracy. The time horizon was 32 time slots of 15 minutes each. The system is initially empty, the activities are created from randomly uniformly distributed time windows and energy demand values (earliest $[0,20]$, duration $[4,10]$, demand $[5,10])$. The main parameter to determine the runtime performance is number of activities $\mathrm{A}$. $\mathrm{M}$ and $\mathrm{P}$ are selected accordingly to provide similar load conditions. Table 6 shows the results with the weighted objective (1) with $\alpha=0.5$.

Furthermore, we have investigated the impact of the available power $P$ on the solution for both the ILP and heuristic algorithm. Relaxing the constraint $\mathrm{P}$ both solutions slightly improve.

The computations reveal that the solutions obtained with the ILP model, using the CPLEX solver are superior both in accuracy and runtime, see Table 6 . The computational complexity of the local ratio heuristic depends mainly on the number of instances which is proportional to the number of activities. The number of generated instances depends on the input, because a large time window will create more instances than a small one. The runtime depends also on the weight $w$ of instances. The profit of an instance decreases during the first sweep iterations, until it is eventually deleted. Therefore, for small weight values $w(i, j)$ (i.e large $\mathrm{P}$ ), the instances will persist for more iterations, increasing the runtime of the heuristic. The solution quality of the heuristic is in worst case $1 / 2$ of the optimum (for "small" bandwidth activities), as stated in [16] and [15]. Finally, we simulated a varying available power over time (as obtained by real consumption measurements, and by applying a grid flow calculation procedure [5]). The created schedule using the ILP model produces a cumulative load, defined as $L(t)=\sum_{i \mid t \in\left[s_{i}, f_{i}\right]} x_{i} d_{i}$ that is quite tight to the limiting available power profile, see Figure 4.

If we repeat the experiment using the heuris- 


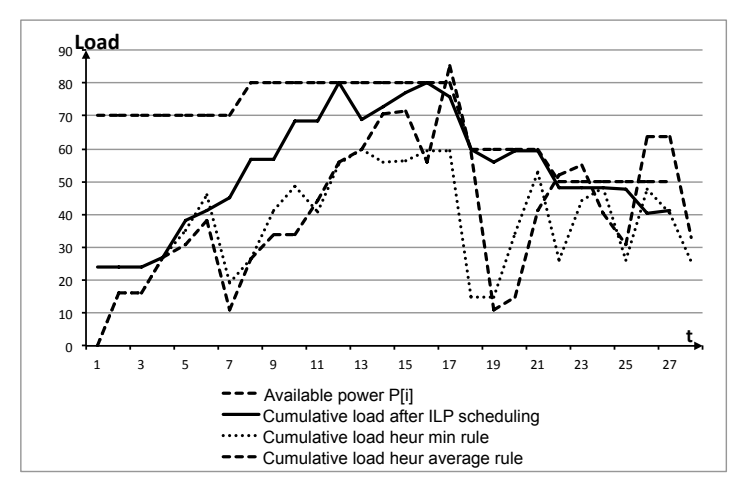

Figure 4: Cumulative load of the optimized activity schedule versus the available power

tic, the performance depends on the calculation of $\bar{w}_{i}$ according to (8) or based on the average value. Using the average, the cumulative load gets closer to the upper limit $P^{t}$, however sometimes it exceeds this limit. Using the minimum rule, the solution is feasible but the performance is poor, see Figure 4.

\section{Conclusions and Future Work}

In this work we addressed two related aspects of e-mobility: the logistic problem of finding a public charging station, and the charging energy balancing problem that affects both the grid provider and the user. We proposed a distributed architecture in which the charging station has some local control autonomy, and which achieves energy balancing at three levels: at the charging station level through controlled charging, at the LV grid level through grid flow calculation and at the routing level using the brokerage function.

We proposed for the user logistic problem an online routing service that uses mobile communication, and by including public city transportation, we have shown that the availability of charging spots can be drastically increased, particularly at low charging station densities.

For the scheduling of charging intervals, we have given an integer linear formulation and a local ratio heuristic and provided preliminary results that show in practice that greedy is too slow and that the accuracy has to be improved. Currently, research is being done to evaluate local search heuristics to the scheduling problem.

The system components, routing server, charging station controllers and low voltage grid flow calculation module are currently integrated with a discrete event simulator that will simulate the routing and energy related processes: activity based mobility model in which EVs issue reservation requests on-trip, routing, arrival and plugin, charging and leaving. Efficiency metrics such as utilization of charging resources, overall performance of different charging strategies and interaction usability are going to be evaluated.

\section{Acknowledgments}

This work is performed within the Austrian project KOFLA "Kooperative Fahrerunterstützung für Lademanagement von elektrischen Fahrzeugen", which is funded by the programme "ways2go" and the Austrian Federal Ministry for Transport, Innovation and Technology. Special thanks to our partners Wiener Stadtwerke and VKW for providing mobility and grid data.

\section{References}

[1] KOFLA Project website, online: http://www.ftw.at/researchinnovation/projects/kofla

[2] M. Herry, R. Tomschy, Travel behaviour survey in Lower Austria 2008, Final Report, Vienna 2009.

[3] M. Herry, R. Tomschy, Travel behaviour survey in Vorarlberg 2008, Final Report, Vienna 2009.

[4] Antti Rautiainen, Corentin Evens VTT, Sami Repo, Pertti Järventausta, Requirements for an Interface Between a Plug-in Vehicle and an Energy System,IEEE PES PowerTech,Trondheim 2011.

[5] A. Schuster, M. Litzlbauer, Easy grid analysis method for a central observing and controlling system in the low voltage grid for E-Mobility and renewable integration, 3rd European Conference SmartGrids and E-Mobility, Munich, October 17-18, 2011.

[6] J. A. Pecas Lopes, F. J. Soares, P. M. Almeida, M. Moreira da Silva, Smart Charging Strategies for Electric Vehicles: Enhancing Grid Performance and Maximizing the Use of Variable Renewable Energy Resources, EVS-24, Stavanger, Norway, May 13-16, 2009.

[7] R.A.Verzijlbergh, Z.Lukszo, J.G.Slootweg, M.D.Ilic, The Impact of Controlled Electric Vehicle Charging on Residential Low Voltage Networks, International Conference on Networking, Sensing and Control Delft, the Netherlands, 11-13 April 2011.

[8] K. Clement, E. Haesen, and J. Driesen, Coordinated Charging of Multiple Plug-In Hybrid Electric Vehicles in Residential Distribution Grids, PES Power Systems Conference and Exposition, 2009. 
[9] O. Sundstrom and C. Binding, Planning Electric-Drive Vehicle Charging under Constrained Grid Conditions, International Conference on Power System Technology, 2010.

[10] P. Sanchez-Martin and Guillermo Sanchez, Optimal Electric Vehicles Consumption Management at Parking Garages, IEEE PES Trondheim PowerTech 2011.

[11] Draft ETSI TS 101 556-1, Intelligent Transport System (ITS); I2V Application; Electric Vehicle Charging Spot Notification Specification, 2011.

[12] CloudMade extract of Wien, Austria from OpenStreetMap.org. Downloaded August 2011.

[13] Wienerlinien, Ein Blick auf Die Leistung Jahresbericht 2010

[14] Dijkstra, E. W. (1959). A note on two problems in connexion with graphs. Numerische Mathematik 1: 269271.

[15] A Bar-Noy, R Bar-Yehuda, A Freund, A Unified Approach to Approximating Resource Allocation and Scheduling, Journal of ACM, 2001

[16] Allan Borodin, David Cashman, and Avner Magen. 2011. How well can primal-dual and localratio algorithms perform?. ACM Trans. Algorithms 7, 3, Article 29 (July 2011), 26 pages. DOI $=10.1145 / 1978782.1978784$

[17] Reuven Bar-Yehuda, Keren Bendel, Ari Freund, and Dror Rawitz. 2004. Local ratio: A unified framework for approximation algorithms. In Memoriam: Shimon Even 1935-2004. ACM Comput. Surv. 36, 4 (December 2004), 422 463. DOI $=10.1145 / 1041680.1041683$

[18] ETSI TS 101 556-1, Intelligent Transport System (ITS); I2V Application; Electric Vehicle Charging Spot Notification Specification, 2011.

[19] Sandford Bessler, Jesper Grønbæk, Max Herry, Andreas Schuster, Rupert Tomschy. Supporting E-mobility Users and Utilities towards an Optimized Charging Service. European Electric Vehicle Congress, EEVC 2011.

[20] Graphserver: The open source multimodal trip planner.

http://graphserver.github.com/graphserver/.

[21] Gosmore:

http://wiki.openstreetmap.org/wiki/Gosmore/.
[22] Schäfer, R.P. and Thiessenhusen, K.U. and Wagner, P. A traffic information system by means of real-time floating-car data. ITS world congress. Volume 2, 2002.

[23] Martin Peter and Daniel Suter. COMPETE Annex 3. Country reports on congestion and delay measurement and their assessment in the EU and the US. Annex 3 section 16.1. Austria. 2006.

[24] Wienerlinien, Ein Blick auf Die Leistung Jahresbericht 2010.

\section{Authors}

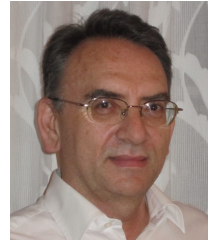

Sandford Bessler has a M.Sc. in Electrical Engineering (1983) and a Ph.D.in Operations Research (1988), both from Vienna University of Technology. From 1980 till 2001 he held different R\&D positions at Kapsch $\mathrm{AG}$ in the development of packet networks, later internet. Since 2001 Dr. Bessler works as a key researcher at The Telecommunications Research Center Vienna (FTW) and as a lecturer at the Vienna University of Technology. His main research interests are resource optimization problems in the e-mobility, and service concepts in intelligent transportation and energy distribution networks.

Jesper Grønbæk has an M.Sc.E.E. (2007) from Aalborg University (AAU). In 2010 he finished his Ph.D. from AAU under the topic of dependable service provisioning in future ubiquitous networks. Since 2010 Dr. Grønbæk has worked as a senior researcher at The Telecommunications Research Center Vienna (FTW). His main research interests are intelligent energy networks, e-mobility and wireless network fault management. 